\title{
Editorials
}

Michael T. Beriault MD FRCPC, J. Roger Maltby MB FRCA FRCPC

\section{The laryngeal mask airway in anticipated difficult airways}

$\mathrm{T}$ HE primary principle of airway management in anaesthesia is to establish and maintain a patent airway to permit effective ventilation. When necessary, airway management also includes provision of a mechanical barrier to pulmonary aspiration of foreign material. The ASA Difficult Airway algorith $\mathrm{m}^{1}$ is predicated on a gold standard of a cuffed endotracheal tube to meet both considerations in every case. This elevates the risk of aspiration to equal priority with effective ventilation, even when no risk factors for regurgitation or aspiration are present. When faced with a difficult airway, the time honoured aphorism of anaesthetists is, "If in doubt, intubate the patient awake."

The correctly positioned laryngeal mask airway (LMA) can provide a clear airway in $99 \%$ of cases. ${ }^{2}$ It protects the lungs from aspiration of foreign material from above its cuff, but not from gastric contents. Therefore, when the threat of aspiration of gastric contents is present, even with an easy airway, use of the LMA is not recommended. The incidence of clinically significant pulmonary aspiration of approximately $l$ in 10,000 in healthy patients undergoing elective surgery is similar for the $\mathrm{LMA}^{2}$ and tracheal intubation or face mask. ${ }^{3}$ If the patient's aspiration risk is low and total lung compliance is near normal, a correctly positioned LMA that allows effective ventilation meets the criterion of a secure airway with minimal risk.

Anaesthetic practitioners have developed a variety of oropharyngeal and nasopharyngeal airway devices during the past hundred years, ${ }^{4}$ including the LMA which became commercially available in the United Kingdom in 1988. Formulation of the ASA Difficult Airway Algorithm began in 1991, but the LMA was not released in the USA until October of that year. This algorithm has had a profound influence on anaesthetists' attitudes and education in the management of the difficult airway. When the algorithm was published in 1993, American experience of the LMA was limited and it did not receive mention. Since then, as anaesthetists' experience has accumulated, the LMA has been promoted from a hands-free substitute for a Guedel airway in short, minor procedures with spontaneous respiration to an alternative to tracheal intubation in selected prolonged, major procedures with positive pressure ventilation. ${ }^{2}$

Few individuals achieve and maintain a full repertoire of strategies and skills with all airway devices. Cormack has pointed out that, because of the rarity of genuinely difficult airway cases, controlled trials are not easy to do and anecdotal evidence can be useful. ${ }^{5}$ Faced with uncertainty, few anaesthetists set aside the collective wisdom of the ASA Difficult Airway algorithm. However, the algorithm implies that tracheal intubation is the preferred management of every airway. Benumof has now indicated five points in the ASA algorithm where the LMA may have a role, ${ }^{6}$ the evidence being based on numerous case reports that attest to its successful use in patients in whom difficult intubation was known or anticipated. Intubation is either a success or failure, and failure leads to alternative second choices of mask ventilation, surgical airway, regional anaesthesia or cancellation of surgery. 'Difficult airway' is an umbrella term which does not distinguish among situations as disparate as unsatisfactory face mask fit, difficult direct laryngoscopy, and pathological or congenital distortion of the larynx or trachea. Widespread experience with the LMA has challenged the practice of routine intubation in elective, healthy patients with normal and difficult airways.

In this issue of the Journal, Giraud $e t a l .^{8}$ report a series of nine patients in whom they used the LMA as the initial airway device in elective patients with recognised predictors of difficult intubation, but no risk fac- 
tors for pulmonary aspiration. All had received surgical and radiation therapy for head and neck malignancy. Limited neck extension was present in the four who had received cervical radiotherapy (modified Mallampati grades I-III), and mouth (interincisor) opening was 18 $\mathrm{mm}$ in the five who had received oral radiotherapy (all grade IV). Two of the latter group had both problems. Transtracheal jet ventilation was immediately available for all cases and, for the patient with mouth opening of $5 \mathrm{~mm}$ who had refused tracheostomy under local anaesthesia, the surgeon was scrubbed and standing by. Following preoxygenation and intravenous induction of general anaesthesia, mask ventilation was easy in all patients. Patients with limited mouth opening then received vecuronium $0.1 \mathrm{mg} \cdot \mathrm{kg}^{-1}$. Insertion of the LMA was difficult in three patients, in two of whom an ingenious lateral insertion technique was used, and ventilation was satisfactory in all five. Conversely, insertion was easy in the four patients who had received cervical lymph node radiation, but ventilation was "inadequate" in two, in whom tracheal intubation was performed under direct laryngosopy, and "difficult" in two others, despite addition of muscle relaxants. The authors confirmed placement of the LMA in front of the glottis by fibreoptic examination, and observed post-radiation oedema and laryngeal sclerosis. These radiation-induced changes did not fully explain their LMA failures because no patient had upper airway obstruction before or after anaesthesia. The authors concluded that their series defined a subset of difficult airways due to cervical irradiation in which the LMA should be avoided.

Are there alternative explanations of these LMA failures? The anaesthetists had moderate to extensive experience of the LMA and different sizes of LMA were tried. Alternative insertion techniques including partial LMA inflation, external compression of the pyriform fossae, anterior traction of the tongue, and reverse insertion with rotation were not mentioned, ${ }^{9}$ and the LMA was seen to be positioned over the glottis. The possibility of reflex glottic closure under light anaesthesia, a common cause of temporary airway obstruction with the LMA, was addressed by the use of neuromuscular blocking agents. In each failure, the true vocal cords could not be seen on fibreoptic laryngoscopy and we agree with the authors that the failures were probably due to a combination of radiation induced changes and inflation of the large LMA cuff in a narrowed hypopharynx.

The importance of this report is that the authors considered the LMA to be a legitimate 'entry point' into the ASA algorithm rather than a rescue device. In similar cases, we prefer to secure the airway with the patient awake and confirm correct placement before inducing general anaesthesia and neuromuscular block- ade, a strategy which respects the primary principle of the ASA algorithm. This study should encourage anaesthetists to consider a role for the LMA in elective patients with recognised difficult airways. It should be used regularly for a wide variety of elective surgical procedures $^{2}$ in patients with normal airways. Only then will anaesthetists have confidence to use it when faced with a difficult airway in similar procedures.

Can we expect success in patients in whom a difficult airway is anticipated? In a personal series of 1500 patients Brimacombe ${ }^{10}$ had no failures in 102 Mallampati III or 13 Mallampati-Samsoon-Young Class IV airways, although he had six failures $(0.4 \%)$ in Mallampati grade I or II airways. Three occurred in the first 250 cases, one in the next 250 , and only one in the next 1000 . In a much larger series, although Mallampati grades were not recorded, Verghese and Brimacombe reported a success rate of LMA insertion at $99 \%$ in 11,910 patients. ${ }^{2}$

The original ASA Difficult Airway Algorithm is an invaluable guide, though it could not, nor did it attempt to, address the management of all varieties of difficult airways with all possible devices. It forced anaesthetists to accept that difficult airways can be foreseen and, with effective strategies, reduce morbidity and mortality. This was progress, and we all like progress. Use of the LMA as an alternative to intubation, even in selected cases, represents a new approach, and some of us do not like change. It is therefore encouraging to know that the ASA Ad Hoc Committee on Practice Guidelines has called for a formal review of the ASA Difficult Airway Algorithm and that the role of the LMA will be one important issue (Caplan RA.: personal communication).

Few anaesthetists devote their clinical practice to surgical centers where similar types of difficult airway problems are encountered often enough to report a series. Those, like Giraud et al., who have that opportunity do us all a service by sharing their experience. The ASA Difficult Airway Algorithm will be enhanced as its underlying principles incorporate new airway devices.

\section{Le masque laryngé et la perspective de l'intubation difficile}

En anesthésie, le premier principe de la maitrise des voies aériennes consiste à établir et maintenir les voies aériennes ouvertes dans le but de ventiler efficacement. Lorsque nécessaire, la gestion des voies aéri- 
ennes impose l'installation d'un écran solide contre l'aspiration de matériel étranger par le poumon. L'organigramme décisionnel de l'ASA devant une intubation difficile prévue (ASA Difficult Airway Algorithm $^{1}$ ) s'appuie sur une règle d'or : en tout temps, la canule endotrachéale avec manchette remplit ces deux exigences. Ceci accorde au risque d'aspiration la même importance que la capacité de ventiler, même en l'absence des facteurs qui favorisent la régurgitation ou l'aspiration. Confrontés à des voies aériennes difficiles, les anesthésistes mettent en pratique l'adage consacré par le temps qui édicte qu'en cas de doute, il faut intuber le patient éveillé.

Le masque laryngé (ML) installé correctement assure la perméabilité des voies aériennes dans $99 \%$ des cas. ${ }^{2}$ Il protège les poumons de l'aspiration de matériel étranger venant de l'étage supérieur mais non du contenu gastrique. Donc, s'il existe un risque d'aspiration du contenu gastrique, et même si l'abord aux voies aériennes est facile, il n'est pas recommandé d'utiliser un ML. L'incidence de l'aspiration pulmonaire cliniquement significative se situe à environ 1 sur 10000 patients bien portants en chirurgie réglée ; elle ne differe pas, que ce soit sous $\mathrm{ML}_{,}{ }^{2}$ intubation endotrachéale ou masque facial. ${ }^{3} \mathrm{Si}$ le risque d'aspiration est faible et que la compliance pulmonaire totale est à peu près normale, un ML placé correctement et capable de procurer une ventilation efficace, rencontre le critère de l'abord aérien protégé sans présenter de risque substantiel.

Au cours des dernières années, les anesthésistes se sont enrichis d'une grande variété de canules oro- et nasopharyngées, ${ }^{4}$ dont le ML qui fut commercialisé au Royaume-Uni en 1988. La formulation de l'organigramme de l'ASA devant des voies aériennes difficiles a débuté en 1991, mais le masque laryngé n'a fait son apparition aux USA qu'en octobre de cette année-là. Cet organigramme a profondément influencé les attitudes et la formation des anesthésistes vis-à-vis les voies aériennes difficiles. Quand l'organigramme a été publié en 1993, l'expérience américaine du ML était limitée et on en faisait peu mention. Depuis, avec l'expérience, le ML, parce qu'il libère les mains, non seulement remplace la canule Guedel mais constitue une alternative à l'intubation trachéale pour des interventions majeures choisies, de durée prolongée, nécessitant la ventilation mécanique. ${ }^{2}$

Peu d'individus deviennent et demeurent compétents et habiles avec toutes les méthodes d'accès aux voies aériennes. Comme l'a énoncé Cormack, les voies aériennes réellement difficiles sont rares, ce qui empêche la réalisation d'études contrôlées et nous oblige à nous appuyer sur des comptes rendus anecdotiques. ${ }^{5}$ En face de l'inconnu, peu d'anesthésistes, confrontés à une intubation difficile, mettent de côté l'organigramme décisionnel de l'ASA, un produit de la sagesse collective. Cet organigramme favorise l'intubation trachéale pour le contrôle des voies aériennes. Benumof désigne maintenant cing situations dans l'organigramme où le ML pourrait jouer un rôle, ${ }^{6}$ sur la base de nombreuses observations confirmant sa valeur dans des situations où des difficultés d'intubation sont connues ou anticipées.? On réussit ou on échoue l'intubation ; l'échec nous oblige à substituer des alternatives de deuxième choix comme la ventilation au masque, l'approche chirurgicale, l'anesthésie régionale ou l'annulation de l'intervention. L'abord difficile constitue un terme général qui ne fait aucune distinction entre des situations aussi disparates que l'inadaptabilité du masque facial, la laryngoscopie directe difficile ou les déformations pathologiques ou congénitales du larynx ou de la trachée. La vaste expérience vécue avec l'organigramme du ML a mis en doute la pratique de l'intubation systématique de patients bien portants aux voies respiratoires normales ou difficiles dans des situations non urgentes.

Ce mois-ci, dans le Journal, Giraud $e t a l^{8}$ rapportent une série de neuf patients électifs pour lesquels ils ont choisi au départ d'aborder les voies aériennes avec le ML ; ces patients avaient des prédicteurs reconnus d'intubation difficile mais aucun facteur de risque d'aspiration pulmonaire. Tous avaient été opérés ou irradiés pour des cancers de la tête ou du cou. L'extension du cou était limitée chez les quatre patients traités avec de la radiothérapie cervicale (échelle de Mallampati modifiée grades I-III) et cinq patients qui avaient reçu de la radiothérapie buccale (tous grade IV) avaient une ouverture de la bouche (espace interincisives) égale ou inférieure à 18 $\mathrm{mm}$. Deux des patients du dernier groupe associaient les deux problèmes. La ventilation transtrachéale par jet était disponible instantanément dans tous les cas, et pour un patient dont l'ouverture buccale était de $5 \mathrm{~mm}$ et qui avait refusé la trachéotomie sous anesthésie locale, un chirurgien était prêt à intervenir immédiatement. Après oxygénation et induction de l'anesthésie générale par la voie intraveineuse, tous les patients ont été ventilés au masque facilement. Les patients dont l'ouverture buccale était limitée ont par la suite reçu $0,1 \mathrm{mg} \cdot \mathrm{kg}^{-1} \mathrm{de}$ vécuronium. L'insertion du ML a été laborieuse chez trois patients, chez deux desquels une insertion latérale a été utilisée. Les cinq ont été ventilés efficacement. À l'inverse, l'insertion a été facile chez les quatre patients qui avaient eu une irradiation de ganglions lymphatiques cervicaux, mais la ventilation a été inefficace chez deux patients qu'il a fallu intuber sous laryngoscopie directe, et difficile chez les deux autres malgré l'ajout de myorelaxants. Les auteurs ont confirmé la position antérieure à la glotte du ML par examen fibroptique et ont observé de l'oedème postirradiation et de la sclérose laryngée. 
Ces mutilations induites par les radiations n'expliquent pas complètement les échecs du ML parce qu'aucun patient n'a présenté d'obstruction des voies respiratoires supérieures avant et après l'anesthésie. Les auteurs ont conclu que leur série délimitait un sous-groupe d'accès aux voies aériennes difficiles causés par l'irradiation cervicale pour qui le ML devrait être évité.

Les carences du ML s'expliquent-elles autrement ? Les anesthésistes possédaient une expérience modérée à étendue du ML et ils ont essayé des ML de différentes tailles. Ils ne mentionnent pas les techniques alternatives d'insertion dont le gonflement partiel du ballonnet du ML, la compression externe de la fosse piriforme, la traction antérieure de la langue et l'insertion inversée avec rotation, ${ }^{9}$ et ils ont constaté la position supraglottique du ML. Ils ont abordé avec l'administration d'un myorelaxant le problème de la fermeture réflexe de la glotte sous anesthésie légère, une cause fréquente d'obstruction transitoire des voies aériennes supérieures avec le ML. Dans chacun des échecs, les cordes vocales ne pouvaient être visionnées sous fibrolaryngoscopie et nous sommes d'accord avec les auteurs pour affirmer que ces échecs étaient vraisemblablement causés par une association de changements induits par les radiations et du gonflement du volumineux ballonnet du ML à l'intérieur d'un hypopharynx étroit.

L'importance de ce compte rendu repose sur le fait que les auteurs ont considéré le ML comme une incursion légitime dans l'organigramme de l'ASA plutôt qu'un simple dispositif de sauvetage. Dans ce type de cas, nous préférons assurer chez un patient éveillé la perméabilité des voies aériennes et confirmer la bonne position de la canule endotrachéale avant l'induction de l'anesthésie généralc et la curarisation, une stratégie conforme aux principes primordiaux de l'organigramme de l'ASA. Cette étude devrait encourager les anesthésistes à considérer un rôle pour le $\mathrm{ML}$ en chirurgie non urgente chez des patients reconnus pour avoir des accès difficiles aux voies respiratoires. Le ML devrait être utilisé régulièrement dans une grande variété d'interventions non urgentes ${ }^{2}$ chez des patients dont les voies aériennes sont normales. Alors, les anesthésistes auront la confiance désirée pour l'employer lorsqu'ils seront confrontés à des voies aériennes difficiles pour des interventions de même type.

Pouvons-nous anticiper le succès avec le $M L$ chez des patients dont un abord difficile aux voies aériennes est prévu ? Dans sa série personnelle de 1500 patients, Brimacombe ${ }^{10}$ n'a essuyé aucun échec chez 102 patients Mallampati de classe III ou IV sur l'échelle MallampatiSamsoon-Young, malgré ses six échecs $(0,4 \%)$ dans les classes I et II de Mallampati. Trois de ceux-ci sont survenus dans les 250 premiers cas, un dans les 250 autres et un seul est survenu dans les 1000 cas suivants. Au cours d'une série beaucoup plus importante, bien que les scores de Mallampati n'aient pas été enregistrés, Verghese et Brimacombe font état d'un taux de succès de 99\% chez 11910 patients. $^{2}$

L'organigramme des voies aériennes difficiles de l'ASA représente un guide inestimable, bien qu'il ne pouvait pas et n'essayait pas d'aborder la gestion de toutes les variétés de voies aériennes difficiles avec tous les types d'instruments imaginables. Il a obligé les anesthésistes à réaliser l'éventualité de difficultés d'accès et qu'avec des approches appropriées, ils pouvaient diminuer la morbidité et la mortalité. C'était là un progrès et nous aimons tous progresser. L'emploi du ML représente une alternative à l'intubation et même si dans certains cas choisis, il constitue une approche nouvelle, certains d'entre nous n'aiment pas le changement. Il est donc encourageant de constater que le comité ad hoc de l'ASA sur les lignes de conduite à suivre a demandé une révision en bonne et due forme de l'organigramme des voies aériennes difficiles et que le rôle du ML fera l'objet d'une étude particulière. (Caplan RA : communication personnelle).

Peu d'anesthésistes exercent dans des centres chirurgicaux où se rencontrent des problèmes de voies aériennes en nombre suffisant pour permettre de monter une série de cas. Ceux qui, comme Giraud et al. ont cette opportunité rendent un service à tous en partageant leur expérience. L'organigramme de l'ASA sur les voies aériennes difficiles sera renforcé à mesure que les principes sur lesquels il repose seront applicables au matériel nouveau.

\section{References}

1 American Society of Anesthesiologists Task Force on Management of the Difficult Airway: Practice guidelines for management of the difficult airway: a report. Anesthesiology 1993; 78: 597-602.

2 Verghese C, Brimacombe JR. Survey of laryngeal mask airway usage in 11,910 patients: safety and efficacy for conventional and nonconventional usage. Anesth Analg 1996; 82: 129-33.

3 Warner $M A$, Warner ME, Weber JG. Clinical significance of pulmonary aspiration during the perioperative period. Anesthesiology 1993; 78: 56-62.

4 McIntyre JWR. Oropharyngeal and nasopharyngeal airways: I (1880-1995). Can J Anaesth 1996; 43: 629-35.

5 Cormack RS. Anaesthesia with the difficult airway. Anaesthesia 1997; 52: 704.

6 Benumof JL. Laryngeal mask airway and the ASA difficult airway algorithm. Anesthesiology 1996; 84: 686-99. 
7 Brimacombe JR, Brain AIJ. The Laryngeal Mask Airway. A Review and Practical Guide. London: W.B. Saunders, 1997: 178-99.

8 Giraud $O$, Bourgain $J$, Marandas $P$, Billard V. Limits of laryngeal mask airway in patients after cervical or oral radiotherapy. Can J Anaesth 1997; 44: 1237-41.

9 Dingley J, Asai $T$. Insertion methods of the laryngeal mask airway. A survey of current practice in Wales. Anaesthesia 1996: 51: 596-9.

10 Brimacombe JR. Analysis of 1500 laryngeal mask uses by one anaesthetist in adults undergoing routine anaesthesia. Anaesthesia 1996; 51: 76-80. 